\title{
Neural Activity in the Frontal Eye Fields Modulated by the Number of Alternatives in Target Choice
}

\author{
Kyoung-Min Lee ${ }^{1,2}$ and Edward L. Keller ${ }^{1}$ \\ ${ }^{1}$ Smith-Kettlewell Eye Research Institute, San Francisco, California 94115, and ²Department of Neurology, Seoul National University, Seoul 110-744, Korea
}

\begin{abstract}
Selection of identical responses may not use the same neural mechanisms when the number of alternatives (NA) for the selection changes, as suggested by Hick's law. For elucidating the choice mechanisms, frontal eye field (FEF) neurons were monitored during a color-tolocation choice saccade task as the number of potential targets was varied. Visual responses to alternative targets decreased as NA increased, whereas perisaccade activities increased with NA. These modulations of FEF activities seem closely related to the choice process because the activity enhancements coincided with the timing of target selection, and the neural modulation was greater as NA increased, features expected of neural correlates for a choice process from the perspective of Hick's law. Our current observations suggest two novel notions of FEF neuronal behavior that have not been reported previously: (1) cells called "phasic visual" that do not discharge in the perisaccade interval in a delayed-saccade paradigm show such activity in a choice response task at the time of the saccade; and (2) the activity in FEF visuomotor cells display an inverse relationship between perisaccadic activity and the time of saccade triggering with higher levels of activity leading to longer saccade reaction times. These findings support the area's involvement in sensory-motor translation for target selection through coactivation and competitive interaction of neural populations that code for alternative action sets.
\end{abstract}

Key words: saccade; decision; response selection; macaque; frontal eye fields; single-unit activity

\section{Introduction}

Life is full of choices: some are trivially simple and automated, such as choosing a correct pedal for foot action at a red or green traffic light, whereas others like career choices or spouse selection require long deliberation and multidimensional calculation. All choice processes involve selection of a response among many competing alternatives. Often a particular selection is driven by a cue whose symbolic meaning has been learned before the selection. This makes choice behaviors distinct from random behaviors, which are independent of cues and also from simple reflexive behaviors evoked by perceptual saliency. Instead, choice behaviors are directed by the symbolic meaning of a stimulus derived from pretrained associations between stimuli and responses (Hick, 1952; Teichner and Krebs, 1974).

An important characteristic of choice responses is that the latency of the behavioral response increases in logarithmic proportion to the number of alternatives (NA) available for the choice (Hick, 1952; Teichner and Krebs, 1974). We demonstrated previously that saccadic eye movements also follow this lawful relationship (Hick's law) in a color-to-location choice saccade task (Lee et al., 2005). This temporal property of choice process is not purely perceptual or mo-

Received Aug. 8, 2007; revised Dec. 15, 2007; accepted Jan. 22, 2008.

This work was supported by grants from the National Institutes of Health (EY-08060 to E.L.K.), the SmithKettlewell Eye Research Institute, and the Brain Research Center of The 21st Century Frontier Research Program funded by the Ministry of Science and Technology of the Republic of Korea (K.M.L.). We thank Dr. Byeong-Taek Lee for his help on data analysis and Jessica Hill for her help with animal care and editing figures.

Correspondence should be addressed to Dr. Kyoung-Min Lee, The Smith-Kettlewell Eye Research Institute, 2318 Fillmore Street, San Francisco, CA 94115. E-mail: kminlee@ski.org.

D01:10.1523/JNEUROSCI.3596-07.2008

Copyright $\odot 2008$ Society for Neuroscience $\quad 0270-6474 / 08 / 282242-10 \$ 15.00 / 0$ tor in nature because the sensory stimulus and the motor response are exactly the same for the choice with different NA. Therefore, the increasing response latency must reflect cognitive processes such as modulation of the sensory-to-motor translation, retrieval of pretrained response alternatives, or selection of the correct response from the alternatives.

The fact that Hick's law is consistently obeyed in wide-ranging situations of choice behavior (Teichner and Krebs, 1974) hints that NA exerts an important influence on choice process. It is unknown, however, how the choice process and NA effects are reflected in neural activity and which cortical regions are involved in these processes. Our recent functional MRI study using human subjects revealed a number of cortical sites where the brain activity was correlated with NA for a choice saccade task (Lee et al., 2006). Among the sites was the frontal eye fields (FEFs), which have been implicated not only in the control of eye movements (Goldberg and Segraves, 1989; Tehovnik et al., 2000), but also higher-level cognitive processes such as selective attention (Kodaka et al., 1997; Kastner et al., 1998; Moore et al., 2003) (but see Goldberg and Bushnell, 1981), target selection (Schall and Hanes, 1993; Thompson et al., 1996; Schiller and Chou, 2000), and motor set (Everling and Munoz, 2000). Here, we studied monkey FEFs to examine the effect of NA variation on neural activity during the same choice saccade task used in the human imaging study.

\section{Materials and Methods}

Subjects and surgical procedures

Two adult male rhesus monkeys (Macaca mulatta) weighing between 6 and $8 \mathrm{~kg}$ were used. Experimental procedures were approved by the 
Smith-Kettlewell Animal Care and Use Committee and were in compliance with the United States Public Health Service Guide for the Care and Use of Laboratory Animals.

A head-restraint post and a stainless-steel recording chamber were implanted under isoflurane anesthesia and sterile surgical conditions. The recording chamber (15 $\mathrm{mm}$ inner diameter) was positioned over a craniotomy centered on the left arcuate sulcus.

\section{Behavioral tasks}

Delayed saccade task. While the monkey fixated at a central, gray fixation point, a single gray target appeared at selected peripheral locations. The monkey was required to hold fixation until the fixation point was turned off. The offset of the fixation point signaled the animal to make a saccade to the peripheral target to receive a liquid reward. The delay between the target onset and the fixation point offset was varied randomly between 400 and $800 \mathrm{~ms}$. The peripheral target was presented at locations of various eccentricity and direction, to map the response field (RF) of each neuron in terms of traditionally defined visual and motor activities of the neuron.

Color-cued choice saccade task. At the beginning of a trial a gray fixation spot appeared at the center of the visual field. While the monkey was holding fixation on the spot, an array of colored potential targets separated evenly in direction from each other appeared in the peripheral visual field. The colored array was illuminated for $400 \mathrm{~ms}$, and then all target alternatives turned gray so that they were no longer distinguishable by color. After a delay randomly chosen between 400 and $800 \mathrm{~ms}$, the gray fixation spot changed into one of the target colors. This color change at the fixation spot was a cue to make a saccade and indicated which target to choose: the animal had to saccade to the target location that previously had the same color as the cue. The mapping between color and location was held constant throughout the training and experiments. The number of alternative targets was one, two, or four within a block of trials, and in the block the correct target location was randomly sequenced among four locations, one in each quadrant. The animal was rewarded only if a saccade occurred within $1000 \mathrm{~ms}$ from the cue onset and the endpoint was within $2^{\circ}$ and $15^{\circ}$ from the correct target in amplitude and direction, respectively.

Figure 1 shows the task for a 4-NA condition. The task was similar for the other NA conditions except that for the 2-NA condition either the red and green pair of targets or the blue and gold pair was shown, and for the 1-NA condition, only one colored target alternative was shown in each trial.

The fixation spot and the targets were disks of $1^{\circ}$ in diameter When the locations of the potential targets were more than $10^{\circ}$ from the fovea, larger disks of one and a half degrees in diameter were used as targets to enhance the visibility. The eccentricity of targets was set based on the location of the RF of the neuron being recorded. One of the potential targets was centered near the maximally responsive part of the field. The colors used for the targets and the fixation spot were equiluminant and chosen based on the CIE $1976\left(\mathrm{~L}^{*} \mathrm{a}^{\star} \mathrm{b}^{*}\right)$ space, which is approximately uniform in perception of color difference (Wyszecki and Stiles, 1982), such that the colors were at the same distance in the space from the two neighboring ones and the gray. A chromameter (CS-100; Minolta Photo Imaging, Mahwah, NJ) was used for measuring luminance and chromaticity of the colors. The luminance of all the visual stimuli was $1.24 \mathrm{~cd} / \mathrm{m}^{2}$ presented against a background luminance of $0.12 \mathrm{~cd} / \mathrm{m}^{2}$.

In this task, visual, cognitive, and motor processes are separated in time and in space, and the task is ideal for observing internally generated neural signals in isolation from the other processes: (1) because candidate targets appeared (a visual event) well in advance of the actual selection of a target (a cognitive process), the two were separated in time; (2) the cognitive process and a motor process in the task were separated in space because the cue for target selection was given at the fovea while a saccade was directed to a peripheral target. Although we refer to a cognitive process, successful completion of the task involves at least two cognitive processes: accessing the associative memory of a particular color and spatial location and target selection or motor planning. Our task does not allow us to distinguish between these various cognitive

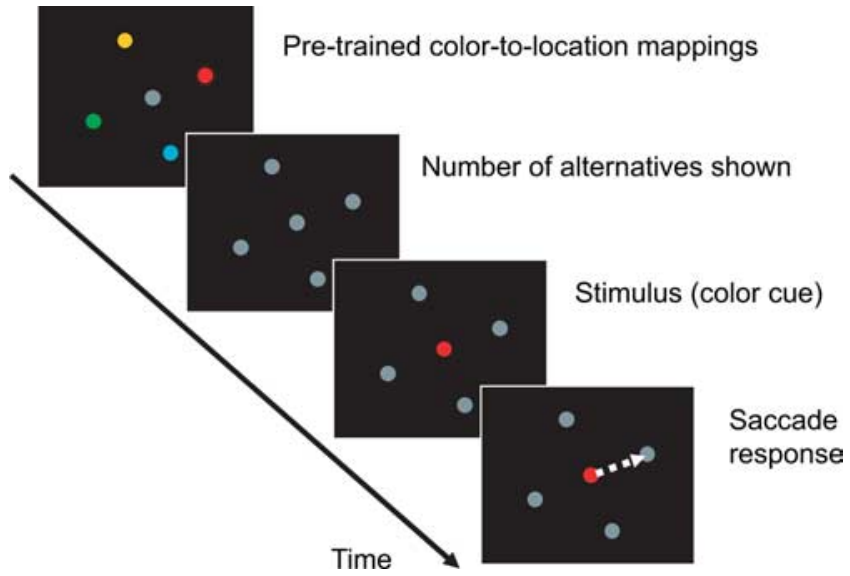

Figure 1. Events in the 4-NA choice saccade task are shown. Color-location associations on which monkeys were trained before recording sessions in the FEF were shown as a reminder of the associations at the beginning of each trial. The alternative array also indicated how many alternatives are available for the trial. The colors of the alternatives were then turned to gray, rendering them indiscriminate at the time of the cue onset. After a further delay, the cue shown at the center was one of the alternative colors presented shown in the array at the beginning of the trial. The monkey was required to make a saccade to the target location that was associated with the cue color, for a liquid reward. One- and two-NA choice trials were similar except that the alternative target array contained only one or two targets, instead of four as shown here.

processes, but does produce a clear cue aligned modulation of activity in many FEF cells.

Note that all potential targets were identical and gray when the cue was given, so that the target selection had to be made by recalling which of the peripheral locations was associated with the color now presented in the cue, and not by a visual matching between cue and targets. Because the association of color and target locations was pretrained and fixed through all blocks of trials, the monkeys could perform the task relying on a long-term symbolic association between color and target location. Therefore, even though the location of the target colors were shown at the beginning of each trial (see Fig. 1), a visual working memory of target configuration was not crucial for performing the task.

Two monkeys were trained on the choice saccade task, each for a period of $\sim 6$ weeks. During the training period alternative blocks of trials with a fixed NA were used, and the red target was usually located in a direction $45^{\circ}$ into the upper-right visual quadrant with the yellow target in the upper-left, the green target in the lower-left and blue target in the lower-right quadrants. However, during each training session the monkey was given training experience with plus or minus spatial rotations of the colored target locations. We found that learning (association of a particular color presented at the fovea with a particular spatially directed saccade) at the nominal $45^{\circ}$ oblique location transferred well to other oblique locations as long as the target color did not cross nominal quadrant boundaries (e.g., the red target remained in the upper right quadrant). Target array eccentricity was varied during training between 5 and $15^{\circ}$. We adopted this training regimen so that once recording of cells began we could rotate and size the eccentricity of the array of potential targets to have one target located near the center of the RF of a particular cell and the animals would still make the color-to-location associations correctly.

\section{Single-cell recordings}

Testing was performed in a dimly illuminated room. A custom real-time program controlled experimental events and data collection, running on a personal computer with dual Xeon processors and Windows XP equipped with two National Instrument multichannel IO boards. Visual targets were displayed on a screen at a distance of $107 \mathrm{~cm}$ from the animal's eyes. Eye position and velocity were recorded with a video-based eye tracker (Eyelink2; SR Research, Mississauga, Ontario, Canada) and sampled at $500 \mathrm{~Hz}$. Extracellular potentials were recorded using tungsten microelectrodes lowered through the recording chamber within a guide 
tube, which penetrated the dura. When we began to encounter neurons with either visual- or saccade-related activities, or both, based on online monitoring, we recorded from every isolated neuron as the electrode was advanced. A window discriminator detected neural discharges and the times were stored at a resolution of $1 \mathrm{kHz}$, and raw data from the microelectrode potential were recorded simultaneously at a sampling rate of $25 \mathrm{kHz}$. Raw records were also examined off-line to check the consistency of spike shape and amplitude, and to segregate, if present, spikes from different units using a template-matching algorithm.

Once a neuron was isolated, we estimated the location of a single, gray visual stimulus that produced the maximal visual- or saccaderelated response using the delayed-saccade paradigm. The array of alternative targets for the cued-choice task was placed so that one of the alternative targets appeared approximately at the location with the maximal response for the neuron. Given the broad direction tuning of FEF neurons, we typically set the target locations at $45^{\circ}$ from the horizontal and vertical meridians. When the activity of a cell was about the same in two of the four tested locations, other oblique positions were tested to identify the location that induced the strongest visual or presaccadic responses.

In choice saccade tasks, trials with different NAs were presented in blocks. In each block, 10 trials were recorded on each of four target positions (40 trials/block). Blocks of different NAs were randomly sequenced across cells. After finishing 10 trials on each NA and target location, another set of blocks were recorded in reverse order if the cell recording could be maintained.

During initial recording sessions, we verified that the penetration was within FEF by micro-stimulation: trains of biphasic pulses with $250 \mu \mathrm{s}$ pulse width and $200 \mathrm{~Hz}$ pulse frequency were delivered for $100 \mathrm{~ms}$ at 100 $\mu \mathrm{A}$ current. If saccades were evoked by the stimulation, lower currents were tested to determine the threshold level at which the probability of evoking a saccade was 0.5 . Penetration sites and depths in which the threshold was up to $50 \mu \mathrm{A}$ were regarded as within the FEF, and only the recordings from such sites and depths were included in the analysis.

\section{Data analysis}

To evaluate the relation between cell discharge and specific events present in the tasks, spike rasters were convolved with a Gaussian kernel $(\sigma=10 \mathrm{~ms})$ to construct spike density curves. A mean spike density function was then generated by averaging the individual spike density curves over trials with the same target condition, aligned with respect to the onset time of targets, the cue, or the saccade. Only correct trials were included in the analysis.

Classification of neurons based on responses in the delayed saccade task. Cells were classified as visual, visuomotor, and motor, based on their activity pattern during the delayed saccade task. This method of classification varied from the original method of Bruce and Goldberg (1985). In their study of FEF cells, they used a learned saccade task to classify motor cells that discharged with saccades in the absence of any visual target. In contrast, cells that were either reliably excited or suppressed by peripheral visual stimuli presented in a no-saccade task were called visual neurons (Bruce and Goldberg, 1985). The method of classifying FEF cells has evolved somewhat since the time of this original work (Bruce and Goldberg, 1985; Joseph and Barone, 1987; Segraves and Goldberg, 1987; Hanes et al., 1998; Sommer and Wurtz, 2001). Our method most closely resembles those of Joseph and Barone (1987) and Hanes et al. (1998). The use of a memory guided saccade paradigm may be better to separate tonic visual and visuomotor cells (Segraves and Goldberg, 1987), but in our study we compare activities in the delayed saccade task with activities in the saccade choice task. These two latter tasks are more alike in terms of the decisions that must be made by the animals.

Cell activity in the delayed saccade task during a $50 \mathrm{~ms}$ interval before the target array onset was regarded as the baseline for our test of visual responses. The activity between 25 and $175 \mathrm{~ms}$ after the target onset was then compared with the baseline. A 50-ms-long window was moved over this period in $10 \mathrm{~ms}$ increments. If the firing frequencies were significantly higher than the baseline for three successive windows, or for a 30 $\mathrm{ms}$ period (Kruskal-Wallis one-way ANOVA, $p<0.01$ for each test, the threshold being one fifth of 0.05 because each time bin was tested five times as the $50 \mathrm{~ms}$ window moved in $10 \mathrm{~ms}$ increments), the cell was classified as exhibiting a visual response. For a test of motor activity, the baseline was measured during the $50 \mathrm{~ms}$ interval preceding the target onset. A $50 \mathrm{~ms}$ time window for comparing the cell activity with the baseline was moved from $200 \mathrm{~ms}$ before to $50 \mathrm{~ms}$ after saccade initiation in $10 \mathrm{~ms}$ increments. Three successive tests had to be significant $(p<$ 0.01 for each Kruskal-Wallis ANOVA test) for a cell to qualify as having a motor response. Neurons with both visual and saccade-related responses were classified as visuomotor.

Detection of an activity modulation by NA. Spike density functions obtained during the choice saccade task were compared across different NA (Kruskal-Wallis one-way ANOVA test on three NA levels). NA effects were tested in three event periods: visual period (from 50 to $250 \mathrm{~ms}$ after the appearance of the array of alternative targets with trials aligned with respect to the visual onset), postcue period (from 50 to $300 \mathrm{~ms}$ after the onset of a cue with trials aligned with respect to the cue onset), and perisaccade period (from $200 \mathrm{~ms}$ before to $50 \mathrm{~ms}$ after saccade initiation with trials aligned with respect to the saccade onset).

\section{Results}

Behavioral results obtained in two monkeys while collecting neural data with the choice saccade task (Fig. 1) were consistent with our previous finding that saccade latency increased monotonically with NA in human subjects using an identical task(Lee et al., 


\section{Saccade direction}

\section{Toward RF .}

\section{Away from RF s.}

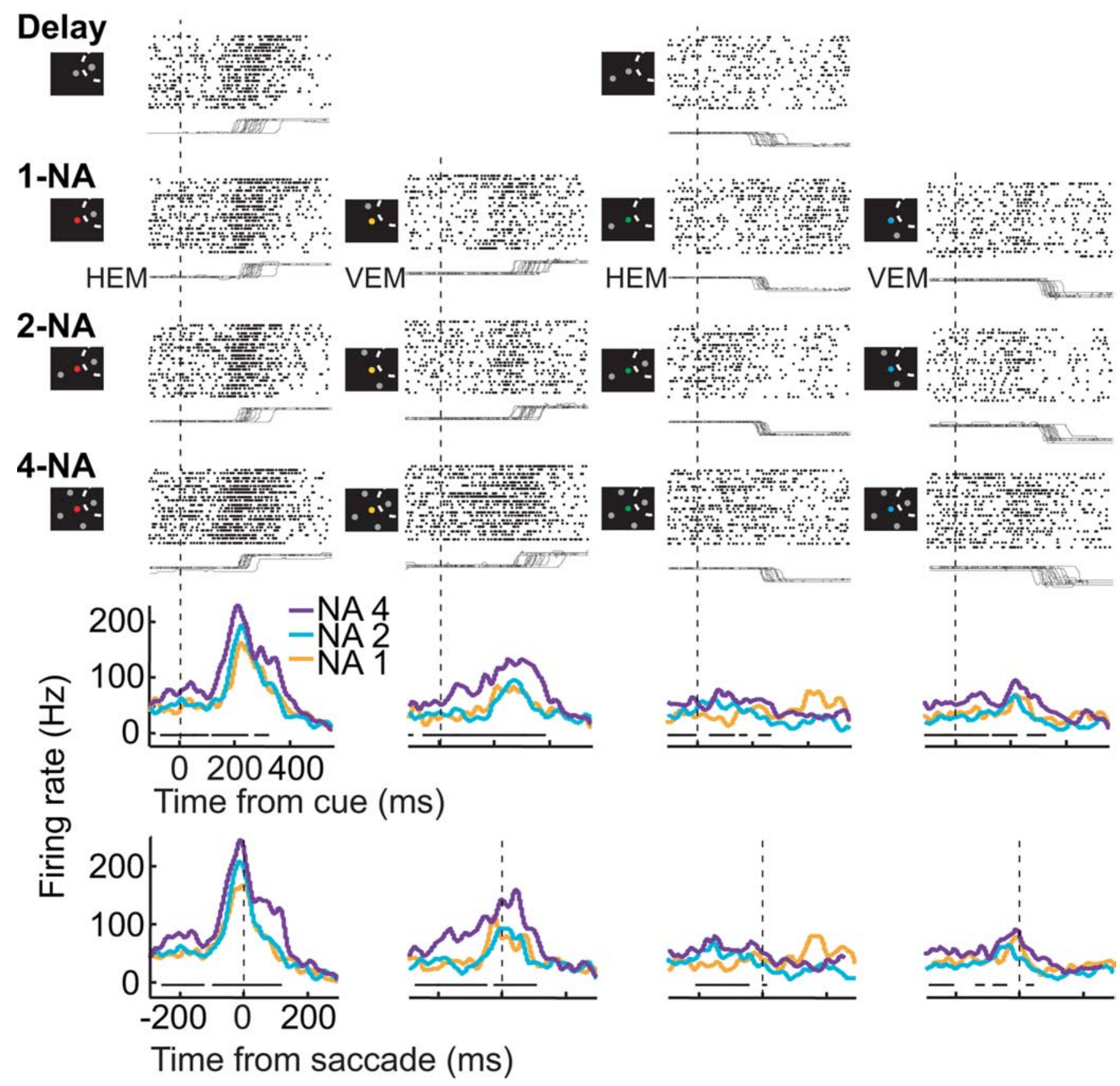

Figure 3. Changes in activity of a visuomotor neuron are shown for different saccade directions and NA conditions. Data shown in raster plots are aligned on cue onset (dashed vertical lines). Bottom traces in each raster plot show either horizontal (HEM) or vertical (VEM) eye positions for that block of trials. The average spike density traces for the various NA conditions are shown in the plots at the bottom. Spike density data are aligned on saccade onset in each lowest plot and on cue onset in the plots second row from the bottom. The black horizontal bars in the two bottom rows of plots indicate the times during the trials when there was a significant difference in activity between the NA conditions.

2005). The mean latency in the delayed saccade task was $201.3 \mathrm{~ms}$, pooled for the two animals, and those in the choice saccade task were 251.2, 271.7, and $284.3 \mathrm{~ms}$ for 1-, 2-, and 4-NA conditions, respectively.

Both monkeys performed the tasks well during the recording sessions: monkey J was correct in $98 \%$ of trials in the choice saccade task and monkey $\mathrm{H}$ was correct in $96 \%$ when NA was four and the array arrangement was at the nominal $45^{\circ}$ rotation. Sufficient correct trials were obtained for all arrangements of the array so that only correct responses are considered in the follow- 
ing analysis. Both monkeys scored close to $100 \%$ in the delayed saccade task and choice saccade tasks with NA of 1 or 2 .

We measured eye movement accuracy in trials conducted with the delay task and with the 1-NA and 4-NA conditions and found no significant differences between any of the conditions ( $p>0.05, t$ test, with Bonferroni corrections for multiple comparisons). In addition, we measured peak radial eye velocity in these same trials and found no significant difference in this parameter between these three task conditions $(p>0.05, t$ test, with Bonferroni corrections for multiple comparisons).

A total of 165 cells were recorded from the FEF while two monkeys performed the choice saccade task. The neurons were classified into visual, visuomotor, and motor types, depending on the presence of visual and/or perisaccadic activities in the delayed saccade task. There were 33 visual, 71 visuomotor, and 36 motor cells. The remaining 25 cells were found by off-line analysis to be uncorrelated with visual and motor events in the delayed saccade task.

\section{NA effect on visual and perisaccade activities in different} cell types

Figure 2 shows the activity of two visual neurons in the choice saccade task. The cell shown in the left column had a transient increase in activity that was aligned with the presentation of the alternative target array. When a single stimulus was shown in the $\mathrm{RF}$ in the 1-NA condition of the choice saccade task, or the delayed saccade task (data not shown), the visual response was greater than when the stimulus was accompanied by alternative targets appearing outside of the RF (2-NA or 4-NA conditions). A similar behavior is demonstrated in another cell in the right column. This neuron had a sustained visual response that gradually declined during the delay period. Comparison between different NA conditions shows that the visual response was much stronger when a stimulus was shown in the RF by itself than when alternative targets were displayed at the same time.

Although a similar decline in visual responses with higher NA was observed in visuomotor cells, activities of the cells when a choice saccade was being made were significantly stronger when choice alternatives were more numerous. An example of this effect of NA on perisaccadic activity is shown in Figure 3. Cell activity after the cue was given at the center was tuned for saccade direction for both the delayed saccade task and the choice saccade task in all NA conditions, and the activity was greater in the 4-NA condition than in the 1- or 2-NA condition. The difference was observed in plots of the spike density function averaged across trials, with trials aligned with respect to saccade onset (the bottom row of panels) as well as to the cue onset (second from the bottom row of panels). Time-points where spike densities were significantly different across NAs are indicated by black bars at the bottom of each panel ( $p<0.05$, Kruskal-Wallis one-way ANOVA test on three NA levels for each $10 \mathrm{~ms}$ bin). The difference was most pronounced when saccades were directed toward the RF (the left-most column), and least when saccade direction was directly away from RF (the third column from the left).

In contrast, perisaccadic activities of motor cells in the FEF for preferred direction were not modulated significantly by the changes in the number of alternatives or between the delayed and choice saccade tasks (Fig. 4). [Although this particular cell exhibited hints of prelude activity in 2- and 4-NA trials along with two more in our sample of 36 motor cells, the prelude activity was not evident in the population analysis (see below), in which all motor cells were pooled together because the number of cells with prelude activity was too small for separate analysis.]

\section{Toward RF}

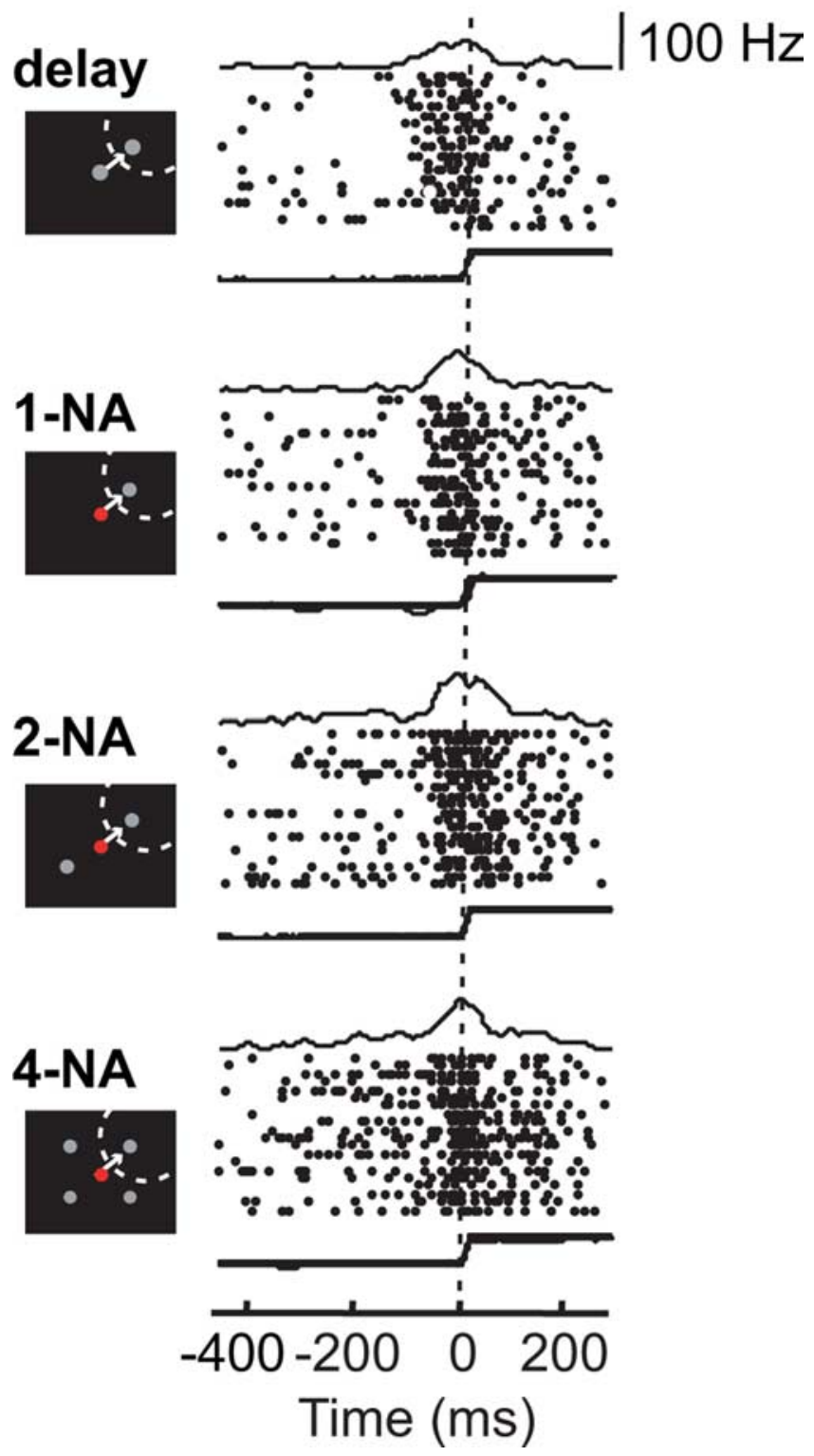

Figure 4. A motor neuron was tested on various NA conditions using our choice saccade paradigm. The perisaccadic activities for saccades toward the RF were invariant despite changes in NA of the choice saccade task. The icons show the viewing conditions after the colored cue was turned on at the fixation point and a saccade is made (zero time on the horizontal axes) to the neutral gray target associated with the cue color. The arrangement of data are the same as in Figure 2 except the traces added at the bottom of each panel show the radial eye positions for each set of trials.

\section{NA effect in the population}

The effect of NA on visual and perisaccadic activities is summarized for all FEF neurons in Figure 5, where spike density rates are averaged over the appropriate time intervals of trials with a saccade directed toward the RF. Temporal averages are compared between 1-NA and 4-NA conditions. In each panel, data for individual cells are marked by circles, with filled ones indicating a statistically significant difference between the two NA conditions $(p<0.05$ in Kruskal-Wallis one-way ANOVA). To test for the presence of NA effect on the population, regression over the data were determined: with an NA effect, the slope of the regression will be significantly different from unity (the dotted lines in the 


\section{Cell type by delayed saccade task}

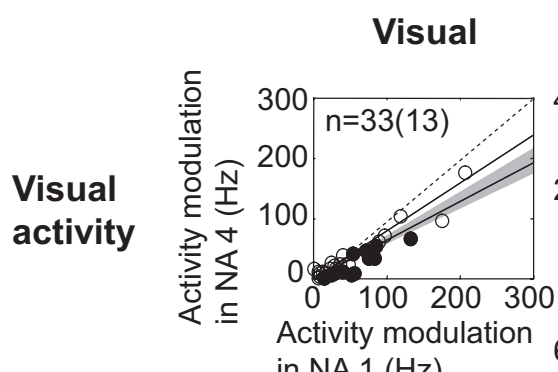

Visuomotor

Motor in $\mathrm{NA} 1(\mathrm{~Hz})$

\section{Post-cue activity}
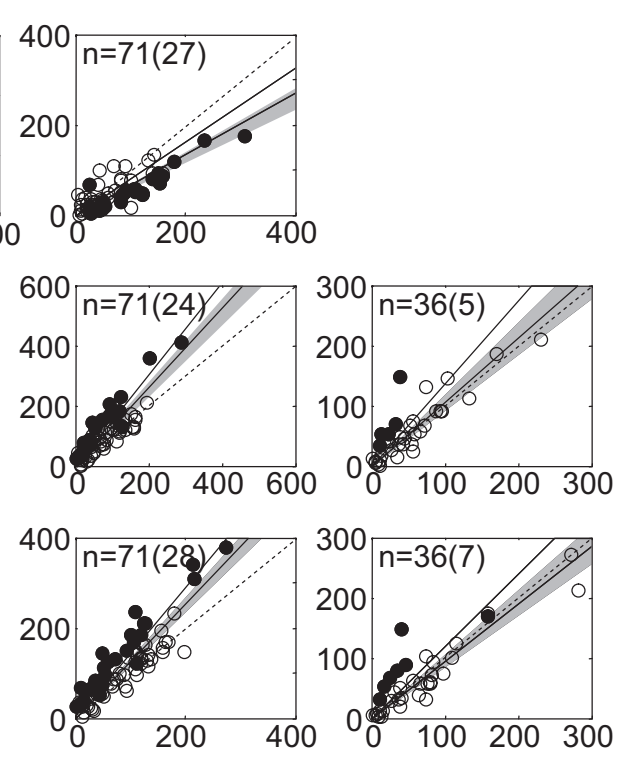

Figure 5. Visual (top row), cue-related (middle row), and perisaccadic (bottom row) responses are compared between 1-NA and 4-NA conditions for visual (left column), visuomotor (middle column), and motor cells (right column) in the FEF. Each circle represents data from an individual neuron. Solid circles indicate that the difference of activity between 1-NA and 4-NA conditions is significantly different ( $p<0.01$, Kruskal-Wallis ANOVA test). The shaded sector in each panel marks the $95 \%$ confidence region for linear regression of 4-NA activity on 1-NA activity in all cells, whereas the sector bounded by two solid lines demarcates that for linear regression of 1-NA activity on 4-NA activity. The dotted lines indicate a relationship with unity slope. The first number in each panel is the total number of cells of that type and the number following in parentheses is the number of individual cells with significantly different activity for the two NA conditions.

figure). Because the regression was between two dependent variables, linear regression was calculated in two ways: 4-NA activity on 1-NA activity, and vice versa. The $95 \%$ confidence regions for the resulting regressions are demarcated, respectively, by the shaded areas and the areas bounded by two solid lines. Both confidence regions lay below the dotted unity line for visual responses of both visual and visuomotor neurons, indicating that the responses in the 1-NA condition were greater than those in the 4-NA condition. The postcue and perisaccade activities of visuomotor neurons had regressions with the slope greater than one, meaning that the activities in 1-NA were smaller than the 4-NA activities. In contrast, the postcue and perisaccade activities of motor cells were not as a group different between 1-NA and 4-NA, with at least one of the regression regions enclosing the unity line.

Similar findings were evident in spike density plots over the neuronal populations (Fig. 6): visual responses were lower in 4-NA than in 1-NA (purple and gold curves in the top row, respectively) for both visual and visuomotor cells (the first and second columns, respectively), whereas the opposite was the case (i.e., higher in 4-NA than in 1-NA) for postcue and perisaccade activities (in the middle and bottom rows, respectively). No NA effect was observed in the population activity of motor cells (the third column). In the figure, thick portions of the curves indicate that the activity is significantly deviated from a baseline, which is chosen as the initial $50 \mathrm{~ms}$ segment in each curve $(p<0.05$ in each $10 \mathrm{~ms}$ bin assuming a Poisson distribution with the mean equal to the baseline activity). Results from statistical tests of NA effect are marked at the bottom in each panel with black bars at the time-points where activities differ significantly among differ- ent NA conditions of the choice saccade task $(p<0.05$ in repeated-design one-way ANOVA).

A few points about the data presented in Figure 6 are noteworthy in comparisons between the delayed saccade condition (black curves) and 1-NA choice saccade task (gold curves). First, the population activities were quite different even though there were many similarities between the two conditions. For example, only one alternative target was available for the saccade response, and the animals needed to suppress a reflexive saccade to the peripheral target. A major difference between the two saccade tasks was in the cueing signal: In the delay task the turning-off of the fixation serves as the go signal to the animal, but specifies nothing about the target. In the choice saccade task the change in color of the fixation point serves as a pointer to the correct target and as the go signal. Second, some cells classified as visual based on the activity in the delayed saccade task exhibited saccade-related responses in choice tasks, especially with higher NAs (the second and third panels in the leftmost column, thick portions of the curves denote a statistically significant rise above the initial baseline, assuming a Poisson distribution, $p<0.05$ ). Analysis of individual neurons revealed significant saccade-related activities in 13 of the 33 traditionally defined visual cells (4-NA condition); examples of such cells are shown in Figure 7. The pauses present in the perisaccadic activity in the cell shown on the right were unusual and were not present in most visual cells with sustained activity.

More remarkably, cells that did not show either visual or saccade-related responses in the delayed saccade task exhibited activities in the choice saccade task (Fig. 6, the second and third panels, right column, unrelated cells) ( 8 of the 25 in an individual analysis of the 4-NA condition). Thus, a classification of FEF neurons using only a delayed saccade task may not reflect true characteristics of some neurons that instead are only revealed by tasks with higher cognitive requirements. Third, motor cells, although lacking a visual response in the traditional sense, showed a significant decline in activity aligned with respect to the appearance of the choice array or the delay target, as shown in the top row and third column in Figure 6.

In addition to the enhancement of postcue and perisaccadic activity in higher NA conditions, visuomotor neurons exhibited another NA effect during the delay period between the onset of the alternative target array and the cue onset. In Figure 8, the population responses aligned on cue onset are shown for trials made to the center of each cell's RF (the $0^{\circ}$ direction) and for trials made to other quadrants $\left(90,180\right.$, and $270^{\circ}$ from the center of the $\mathrm{RF})$. The top row of plots show results for visuomotor cells, and the bottom row motor cells. The first column of plots shows the results for trials made to the center of the fields and, thus, are identical to the plots shown in Figure 6 for visuomotor and motor cells with data aligned on cue onset. Now, notice that the levels of activities for visuomotor cells immediately preceding the cue onset (zero in the plots) differ across saccade directions for 1- and 
2-NA conditions ( $p<0.01$ by repeateddesign one-way ANOVA on saccade direction), but not for 4-NA condition $(p>$ $0.1)$. Thus, the direction-related variation of the precue activity was modulated across NA and, more importantly, these modulations of precue activity coincided very well with the presence or absence of a potential target within the RF: in the 1-NA condition, only the zero direction (the RF of the recorded cells) was where a target was present, and the precue activity was elevated only for this direction (gold curves), whereas in 4-NA trials an alternative target was present in all directions, and so was the elevation of the precue activity (purple curves). In the 2-NA condition, a potential target was located in the RF when the correct target was in either $0^{\circ}$ or $180^{\circ}$, because the two alternative targets were always paired in the opposite directions. No target was shown in the RF when either $90^{\circ}$ or $270^{\circ}$ were the correct direction in $2-\mathrm{NA}$ trials. The direction-variation of the precue activity in 2-NA condition matched exactly with these situations: the activity was high with a target in the $\mathrm{RF}\left(0\right.$ and $180^{\circ}$ directions), and low without (90 and $270^{\circ}$ directions). Note also that over all NA conditions the modulation of the precue activity was binary between two activity levels, presumably reflecting the binary distinction that a target was present or not within the RF of the recorded cells.

In contrast, such modulation of precue baseline activity was not observed in motor cells (Fig. 8, bottom row).

\section{NA effect on direction discrimination in perisaccadic activity}

Given the direction tuning of perisaccadic activity, the timing of direction discrimination was examined; it occurred after a constant delay from the cue onset, but the interval between the onset of the discrimination and the saccade response was variable (Fig. 9). Perisaccadic activity in visuomotor and motor cells were compared between 0 and $180^{\circ}$ directions (with respective to the RF center) and the earliest significant divergence was designated as the discrimination onset (Kolmogorov-Smirnov ANOVA, $p<$ 0.01 for each $50 \mathrm{~ms}$ window moving by $10 \mathrm{~ms}$ increments, three consecutive positive results for qualifying as significant). In Figure 9, the discrimination onsets are plotted as a function of the $\mathrm{RT}$ in the recorded trials. Single dots denote data from individual cells, and the nonvertical lines regressions over the population for each NA condition (indicated by the color). The vertical lines mark the RT for each NA, averaged over all cells. The RT varied much from cell to cell, depending on the training status, motivation levels, etc. of the animals at the time of the recording. Still, the average RT was longer for higher NA, consistent with Hick's law. For this analysis, cells were excluded that had been discriminatory already at the onset of the cue (between 0 and $50 \mathrm{~ms}$ after the cue onset) in 1-NA trials, because the comparison across NA would not be very meaningful in such cases.

In the top, where the discrimination onsets were shown with respect to the cue onset, the slopes of regression were not different from zero ( $p>0.05$ in linear regression for all NAs), indicat-
Cell type by delayed saccade task$$
\text { Visuomotor }
$$$$
\text { Motor }
$$$$
n=71
$$

$n=36$
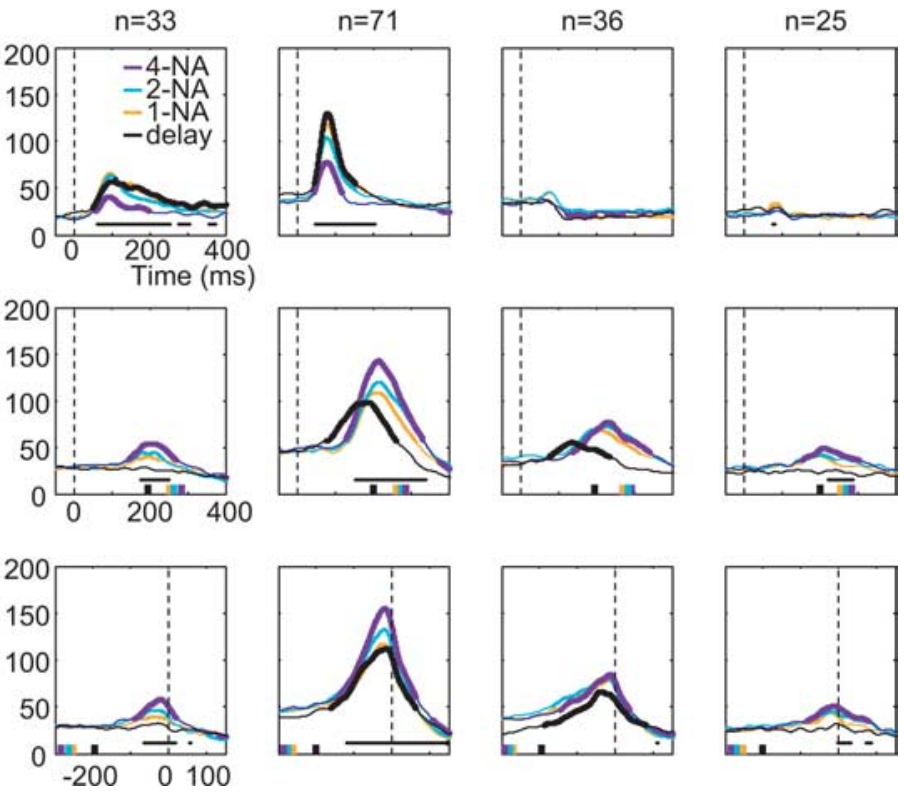

Figure 6. Population activities during the choice saccade task are shown for visual $(n=33)$, visuomotor $(n=71)$, motor $(n=$ 列 small verica aligned on cue onset (middle row) or median time of cue onset (lower row).

ing that the discrimination occurred at a constant delay from the cue, regardless of when the responses were actually initiated and regardless of NA. In contrast, in the bottom where the discrimination times were shown with respect to saccades, the slopes were significantly different from zero $(p<0.05$ in linear regression for all NAs, see the figure caption for the values), indicating that the interval from the direction discrimination in the cell activity to the saccade response was longer in longer RT trials. The regression slopes for different NAs were not different from each other statistically, but there was a tendency that the slope was shallower for higher NA. These comparisons demonstrate that the perisaccadic activities started off by the cue and the neural processing up to the response initiation took longer for more difficult target selection with higher NA.

\section{Discussion}

\section{NA-modulation of FEF activity and target selection}

The frontal eye fields are usually regarded as an oculomotor area involved in generating saccades, smooth pursuit, and fixation (Goldberg and Segraves, 1989; Tehovnik et al., 2000). Recent evidence has also implicated them in higher cognitive functions, such as attention and target selection (Schall and Hanes, 1993; Thompson et al., 1996; Kodaka et al., 1997; Kastner et al., 1998; Everling and Munoz, 2000; Schiller and Chou, 2000; Moore et al., 2003; Sommer and Wurtz, 2004). Current results collaborate further that the area also carries neural signals that reflect target selection: Visual and perisaccadic activities were modulated by NA, a crucial feature of choice process from the perspective of Hick's law.

Behavioral experiments have established that the NA effect is 
A)

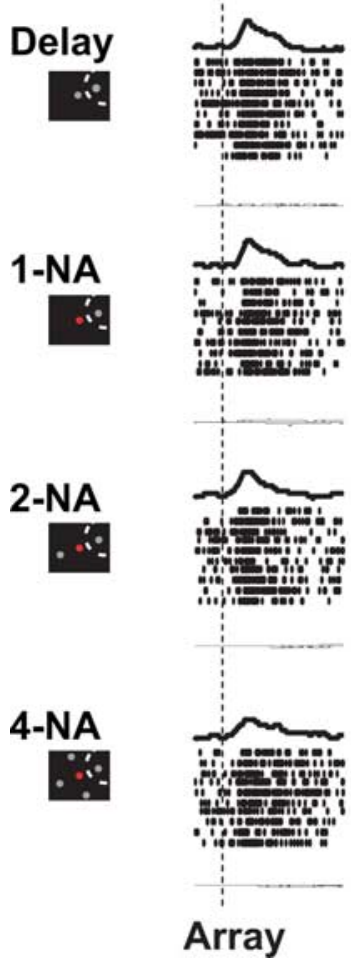

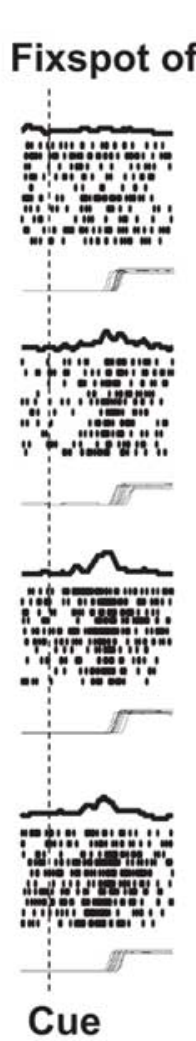

B)

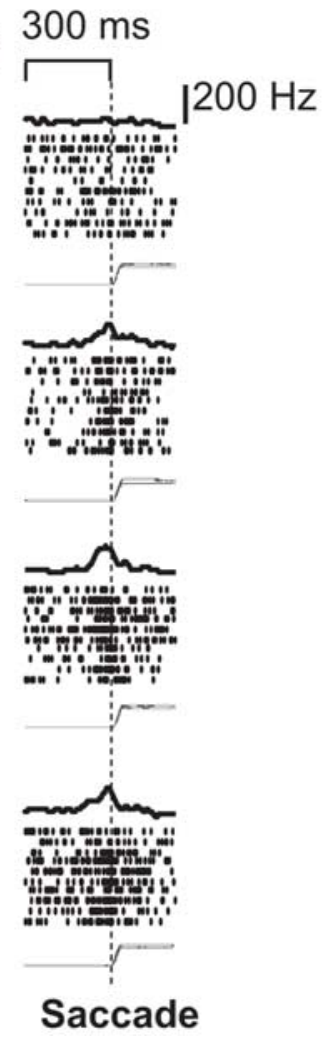

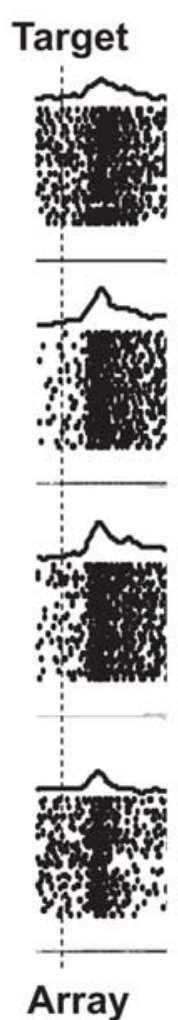

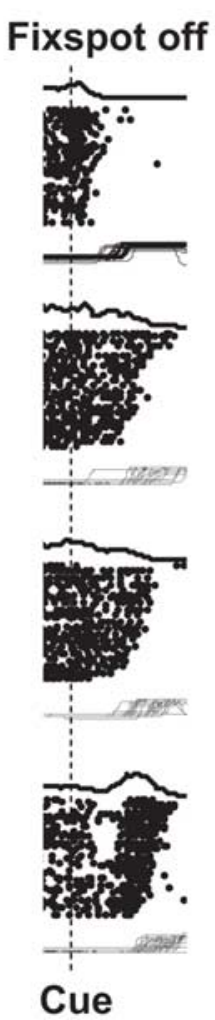

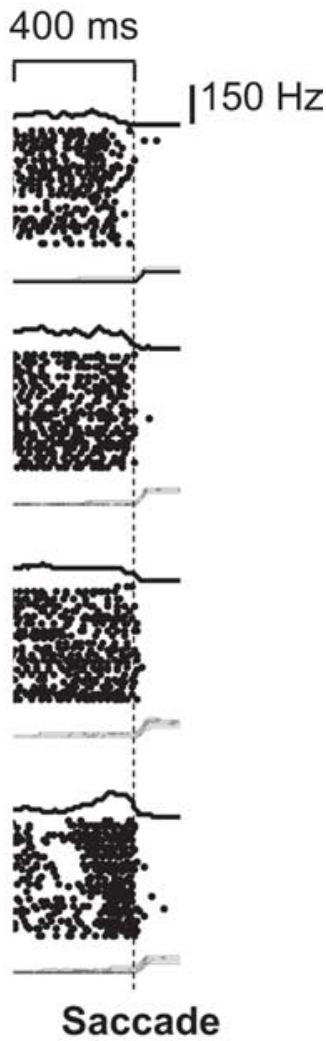

Figure 7. Two cells classified as visual in the delayed saccade task that exhibited significant perisaccadic activities in 4-NA choice trials are shown. The cell in $\boldsymbol{A}$ showed a phasic visual response that dissipated during the delay period, whereas the one in $\boldsymbol{B}$ had a tonic visual activity that lasted until the offset of the fixation spot. The neural activities are aligned with respect to the onset of target array, cue, and saccade in the left, middle, and right columns, respectively. The activities in the delayed saccade task and the choice saccade task with different NAs are shown in the panels of each row. Spike rasters were sorted in a descending order of response times.

predominantly caused by the process of stimulus-to-response transformation, rather than to stimulus encoding or motor preparation/execution (Teichner and Krebs, 1974). Likewise, the observed modulation of FEF activity cannot be attributed to more elementary aspects of the task, such as visual processing of targets/cues, or generating saccades, because they were the same in different NA conditions. The NA-modulation instead suggests that the neural activity of FEF visuomotor cells underlie the choice processes which vary in proportion to the number of choice alternatives. The current neural-recording results obtained in alert monkeys confirm our earlier functional MRI study using human subjects that revealed that the FEF was a cortical site where the brain activity was correlated with NA for a choice saccade task (Lee et al., 2006).

In the majority of visuomotor neurons and in their population activity, visual responses to the appearance of the alternative target array were smaller when the number of potential targets was two or four than when only one target appeared. The decline of visual response suggests that, when only one target appeared, the selection for the ensuing response may have been made at the time of target appearance rather than later at the time of cue onset. In other words, the higher visual activity observed in the 1-NA condition may in fact have included not only responses elicited by direct visual excitation of the RF, but also those related to response selection prompted by the appearance of the solitary target. Similarly, the enhancement of perisaccadic activities in higher NA conditions may have reflected the target selection which could be made only after the cue onset in these conditions.
Thus, the concurrent but opposite modulation of visual and perisaccade activities coincides nicely with the timing of decision making in our choice saccade task, further supporting the view that the FEF activity underlies target choice. It cannot be determined, however, from our current data alone whether the NA modulations in FEF are necessary for making the choice. Such a determination would require future experiments using techniques such as chemical inactivation or surgical lesions to determine the necessity of FEF neurons in generating choice saccades.

Alternatively, the stronger visual response in the 1-NA condition reflects preparation for an ensuing saccade (Goldberg and Bushnell, 1981, 1985), or the modulation might have arisen from lateral inhibition across the visual fields among the visual signals. Additional research will be required to test our idea that the visual modulation is related to target selection against these possibilities.

Previously, Basso and Wurtz (1998) reported that a decrease in a priori probability of different saccade responses decreased the visual activity and the pretarget-selection activity during the delay period in cells in the superior colliculus (SC). We found a similar decrement in some visual and visuomotor neurons in the FEF during the visual response (Figs. 2, 6), but we did not see the probability-based modulation in the pretarget-selection interval in FEF cells (see the level of activity preceding the cue onset in the middle row of plots in Fig. 6). In this pretarget-selection period FEF visuomotor cells showed more activity when an ensuing saccade had a higher probability of being toward their RF (Fig. 8). The FEF cells clearly harbor the a priori information on choice 
alternatives, i.e., whether a saccade will likely be toward their RF or not. However, the generation of a choice saccade does not seem to occur through a threshold mechanism as was proposed for the SC neurons (Basso and Wurtz, 1998).

Dorris and Munoz (1998) also observed enhanced SC activity when the target was more likely to appear in the RF, contrary to our finding in perisaccadic activities of the FEF neurons; the activities were greater with higher NA where the probability of saccade toward the RF was lower. However, we made an observation similar to theirs during the delay period in the choice saccade task; FEF visuomotor cells maintained a higher activity before the cue was presented if a potential target was present within the RF (Fig. 8).

\section{Challenges to traditional notions on FEF neurons}

The traditional method of classifying FEF cells into visual, motor, and visuomotor types (Bruce and Goldberg, 1985; Joseph and Barone, 1987; Hanes et al., 1998; Sommer and Wurtz, 2001) proved too simplistic. Some cells that met the criteria for a visual neuron in the delayed saccade task produced a vigorous perisaccadic activity for choice saccades, and some cells which were classified as nonresponding in the delay saccade paradigm showed a significant perisaccadic response in the choice response task, especially in the 4-NA condition. Thus, although we followed the traditional classification for FEF neurons in this study in the absence of a better idea, we feel that the presence or absence of activity before a saccadic response, which may vary depending on the task requirement, should not be taken as an invariant feature for the classification of FEF cells.

Saccade generation by the FEF has been proposed to include a fixed threshold level which cell activities must reach to trigger the downstream saccade generator in the brainstem (Hanes and Schall, 1996; Everling and Munoz, 2000). Our data does not support this theory. First, in motor cells, the presaccadic activity rose in a manner that was different between the delayed-saccade task and choice saccade tasks. In Figure 6 , the bottom panel in the third column (presaccadic activity, motor cells) shows that in no period before the saccade onset did the activity profiles for delayed saccades and choice saccades come together providing evidence against a threshold mechanism. Second, the presaccadic activity in the visuomotor neurons was consistently higher in higher NA conditions. If a threshold existed for saccade generation, a saccade would have been latency.
Saccade direction (with respect to the center of response field)
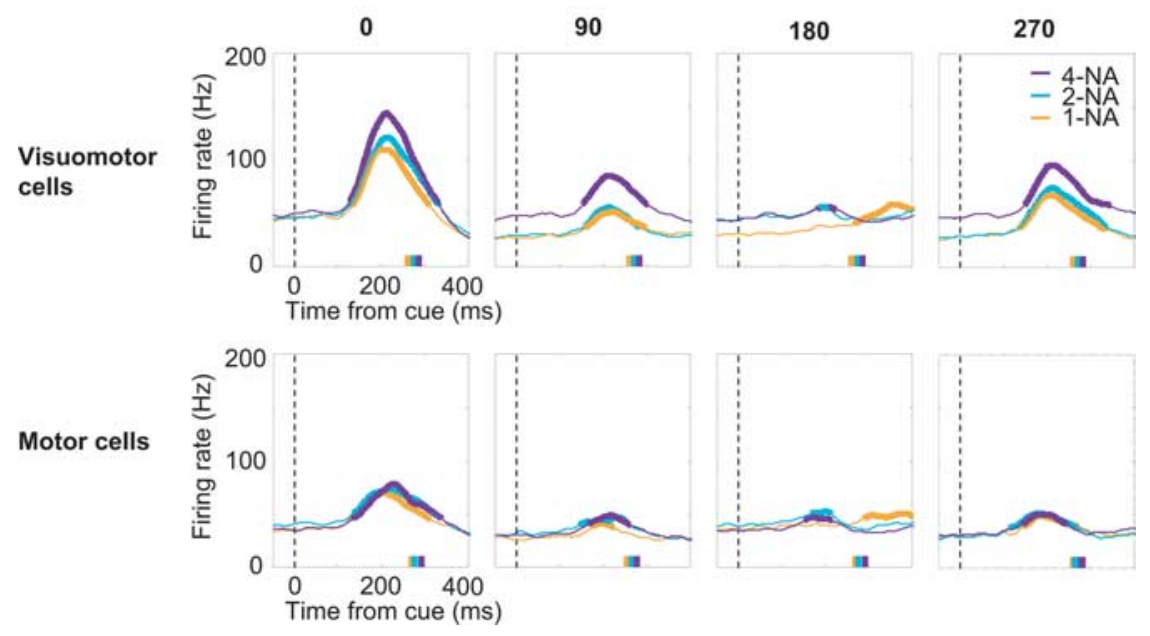

Figure 8. Modulation in the cue-related population activity for visuomotor and motor neurons in FEF as a function of response direction with respect to the direction of the center of the RF. Data is aligned on cue onset (dashed vertical lines). Same color coding scheme for NA condition as in Figures 3 and 6 . The portions of the traces where the activities were significantly different from base line are shown with thickened curves. The small vertical tic marks at the bottom of each plot show the median times of saccade
Visuomotor Cells

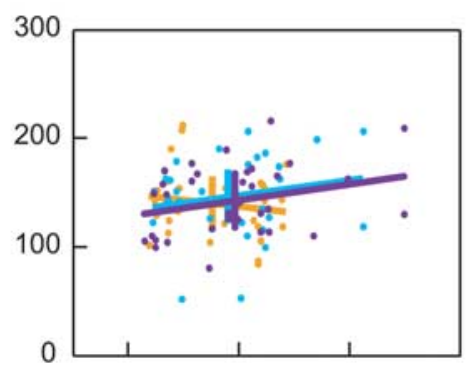

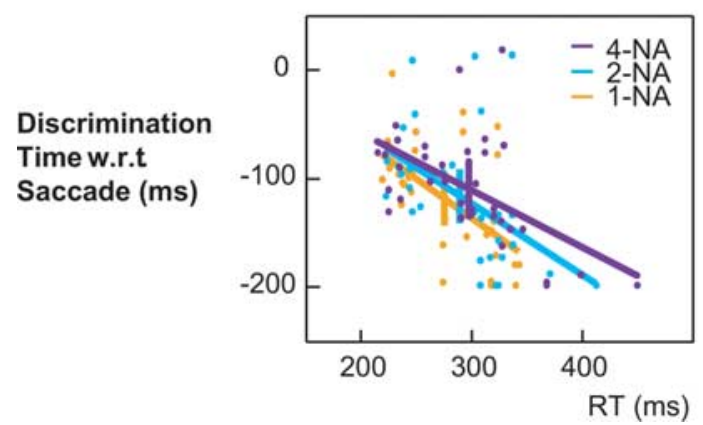

Motor Cells
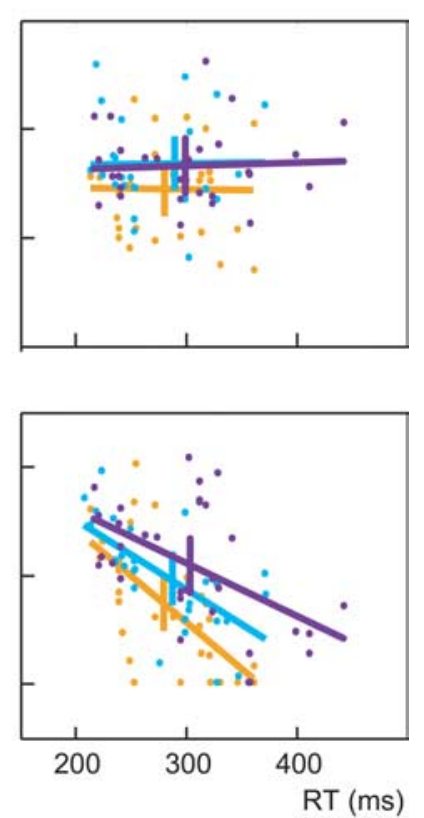

Figure 9. The onset of direction discrimination in perisaccadic activities and mean RT of recorded trials are plotted for individual visuomotor and motor cells. The discrimination times are shown with respect to the cue onset (top) and the saccade response (bottom). Different NA data are denoted by the color. Single dots represent data from individual units, nonvertical lines regressions over the cell population for each NA. Vertical lines crossing the regression lines demarcate the average RT over the population. All regression slopes in the top were not different from zero: the slope values and $95 \%$ confidence intervals were -0.11 $(-0.37 \sim 0.14), 0.14(-0.12 \sim 0.41)$, and $0.15(-0.03 \sim 0.33)$ in 1-, 2-, and 4-NA conditions, respectively, for visuomotor cells, and $-0.02(-0.38 \sim 0.35), 0.01(-0.37 \sim 0.40)$, and $0.03(-0.20 \sim 0.25)$ for motor cells. In contrast, the slopes in the bottom were all significantly different from zero: the slope values and $95 \%$ confidence intervals were $-0.73(-1.1 \sim-0.38)$, $-0.66(-1.0 \sim-0.28)$, and $-0.52(-0.76 \sim-0.28)$ in 1-, 2-, and 4-NA conditions, respectively, for visuomotor cells, and $-0.86(\mu 1.25 \sim-0.47),-0.64(-0.93 \sim-0.36)$, and $-0.49(-0.79 \sim-0.19)$ for motor cells. W.r.t., With respect to.

triggered earlier in higher NA conditions. We found the saccadic latencies to be longer in higher NA conditions. Therefore, rather than fixed with respect to FEF activity, the threshold for triggering a saccade appears to be adjusted by the NA 
information, which is consistent with our previous finding from human saccade latencies using the same choice saccade task (Lee et al., 2005). We suggest that the more rapid increase in presaccade activity in higher NA trials may activate a population of cells elsewhere that elevate the threshold level for triggering a saccade (e.g., the fixation cells in other regions of the FEF and the rostral superior colliculus) (Munoz and Wurtz, 1993; Sommer and Wurtz, 2000).

\section{Increased FEF activity as coactivation of alternative action sets for target selection}

The modulation of activity during the delay period was binary in that the activity was elevated to one level whenever a target was within the RF and maintained at another when no target was within the field (Fig. 8), regardless of NA. However, the modulation of transient activities after the onset of a target array or the cue was graded by NA (Figs. 6,8). The two modes of modulation suggest different functional roles for the modulations.

The delay activity may represent tonic-visual and/or motorpreparatory activities reported previously. We could not distinguish the two possibilities in our experiments, because the memory-guided saccade paradigm was not used. Nevertheless, the binary nature of the activity modulation seems to reflect inclusion or exclusion of an alternative action set, coded by the recorded cell population, for an upcoming target choice.

In contrast, the graded modulation of transient activities may constitute neural processes for choosing one action set over other alternative ones, initiated at the cue onset. Generally the firing rate of a neuron responsive to external events depends on two factors: sensory stimulus and peer inputs from the neural assembly in which the recorded cell is embedded (Harris, 2005). Because the sensory cue (i.e., a color change at the fixation spot) was exactly the same in all NA conditions of our experiments, the increased activity as a function of increasing NA must have come from changes in the input from peer neurons. Regardless of whether the increase in peer input resulted from including a larger population of neurons or enhancing the efficiency of neural transmission within the same population, the onset of an awaited cue led to activation of the prepared neural assemblies to a level that was proportional to NA. Although the details could only be speculated at this point, neural mechanisms whereby cells coding for the correct response won out others that had been coactivated as contenders would most likely involve competitive inhibitory interaction among them.

\section{References}

Basso MA, Wurtz RH (1998) Modulation of neuronal activity in superior colliculus by changes in target probability. J Neurosci 18:7519-7534.

Bruce CJ, Goldberg ME (1985) Primate frontal eye fields. I. Single neurons discharging before saccades. J Neurophysiol 53:603-635.

Dorris MC, Munoz DP (1998) Saccadic probability influences motor preparation signals and time to saccadic initiation. J Neurosci 18:7015-7026.

Everling S, Munoz DP (2000) Neuronal correlates for preparatory set associated with pro-saccades and anti-saccades in the primate frontal eye field. J Neurosci 20:387-400.

Goldberg ME, Bruce CJ (1985) Cerebral cortical activity associated with the orientation of visual attention in the rhesus monkey. Vision Res 25:471-481.

Goldberg ME, Bushnell MC (1981) Behavioral enhancement of visual responses in monkey cerebral cortex. II. Modulation in frontal eye fields specifically related to saccades. J Neurophysiol 46:773-787.

Goldberg ME, Segraves MA (1989) The visual and frontal cortices. In: The neurobiology of saccadic eye movements (Wurtz RH, Goldberg ME, eds), pp 283-313. New York: Elsevier.

Hanes DP, Schall JD (1996) Neural control of voluntary movement initiation. Science 274:427-430.

Hanes DP, Patterson WFI, Schall JD (1998) Role of frontal eye fields in countermanding saccades: visual, movement and fixation activity. J Neurophysiol 79:817-834.

Harris KD (2005) Neural signatures of cell assembly organization. Nat Rev Neurosci 6:399-407.

Hick WE (1952) On the rate of gain of information. Quart J Exp Psychol 4:11-26.

Joseph JP, Barone P (1987) Prefrontal unit activity during a delayed oculomotor task in the monkey. Exp Brain Res 67:460-468.

Kastner S, De Weerd P, Desimone R, Ungerleider LG (1998) Mechanisms of directed attention in the human extrastriate cortex as revealed by functional MRI. Science 282:108-111.

Kodaka Y, Mikami A, Kubota K (1997) Neuronal activity in the frontal eye field of the monkey is modulated while attention is focused on to a stimulus in the peripheral visual field, irrespective of eye movement. Neurosci Res 28:291-298.

Lee KM, Keller EL, Heinen SJ (2005) Properties of saccades generated as a choice response. Exp Brain Res 162:278-286.

Lee KM, Wade AR, Lee BT (2006) Differential correlation of frontal and parietal activity with the number of alternatives for cued choice saccades. NeuroImage 33:307-315

Moore T, Armstrong KM, Fallah M (2003) Visuomotor origins of covert spatial attention. Neuron 40:671-683.

Munoz DP, Wurtz RH (1993) Fixation cells in monkey superior colliculus. I. Characteristics of cell discharge. J Neurophysiol 70:559-575.

Schall JD, Hanes DP (1993) Neural basis of saccade target selection in frontal eye field during visual search. Nature 366:467-469.

Schiller PH, Chou I (2000) The effects of anterior arcuate and dorsomedial frontal cortex lesions on visually guided eye movements: 2. Paired and multiple targets. Vision Res 40:1627-1638.

Segraves MA, Goldberg ME (1987) Functional properties of corticotectal neurons in the monkey's frontal eye field. J Neurophysiol 58:1387-1419.

Sommer MA, Wurtz RH (2000) Composition and topographic organization of signals sent from the frontal eye field to the superior colliculus. J Neurophysiol 83:1979-2001.

Sommer MA, Wurtz RH (2001) Frontal eye field sends delay activity related to movement, memory, and vision to the superior colliculus. J Neurophysiol 85:1673-1685.

Sommer MA, Wurtz RH (2004) The dialogue between cerebral cortex and superior colliculus: implications for saccadic target selection and corollary discharge. In: The visual neurosciences (Chalupa LM, Werner JS, eds), pp 1466-1484. Cambridge, MA: MIT.

Tehovnik EJ, Sommer MA, Chou IH, Slocum WM, Schiller PH (2000) Eye fields in the frontal lobes of primates. Brain Res Reviews 32:413-448.

Teichner WH, Krebs MJ (1974) Laws of visual choice reaction time. Psychol Rev 81:75-98.

Thompson KG, Hanes DP, Bichot NP, Schall JD (1996) Perceptual and motor processing stages identified in the activity of macaque frontal eye field neurons during visual search. J Neurophysiol 76:4040-4055.

Wyszecki G, Stiles WS (1982) Color science: concepts and methods, quantitative data and formulae. New York: Wiley. 